\title{
Crossing numbers of imbalanced graphs
}

\author{
János Pach* \\ Courant Institute, NYU and Rényi Institute, Budapest \\ József Solymosi ${ }^{\dagger}$ \\ University of British Columbia, Vancouver \\ Gábor Tardos T $^{\ddagger}$ \\ Simon Fraser University and Rényi Institute, Budapest
}

\begin{abstract}
The crossing number, $\operatorname{cr}(G)$, of a graph $G$ is the least number of crossing points in any drawing of $G$ in the plane. According to the Crossing Lemma of Ajtai, Chvátal, Newborn, Szemerédi [ACNS82] and Leighton [L83], the crossing number of any graph with $n$ vertices and $e>4 n$ edges is at least constant times $e^{3} / n^{2}$. Apart from the value of the constant, this bound cannot be improved. We establish some stronger lower bounds, under the assumption that the distribution of the degrees of the vertices is irregular. In particular, we show that if the degrees of the vertices are $d_{1} \geq d_{2} \geq \ldots \geq d_{n}$, then the crossing number satisfies $\operatorname{cr}(G) \geq \frac{c_{1}}{n} \sum_{i=1}^{n} i d_{i}^{3}-c_{2} n^{2}$, and that this bound is tight apart from the values of the constants $c_{1}, c_{2}>0$. Some applications are also presented.
\end{abstract}

\section{Introduction}

Let $G$ be a simple undirected graph with $n=n(G)$ nodes (vertices) and $e=e(G)$ edges. A drawing of $G$ in the plane is a mapping $f$ that assigns to each vertex of $G$ a distinct point in the plane and to each edge $u v$ a continuous arc connecting $f(u)$ and $f(v)$, not passing through the image of any other vertex. For simplicity, the arc assigned to $u v$ is also called an edge, and if this leads to no confusion, it is also denoted by $u v$. Assume that no three edges share an interior point. A common interior point of two edges is called a crossing point. The crossing number, $\operatorname{cr}(G)$, of $G$ is the minimum number of crossing points in any drawing of $G$.

The determination of $\operatorname{cr}(G)$ is an NP-complete problem [GJ83]. It was discovered by Leighton [L83] that the crossing number can be used to estimate the

*Supported by NSF Grant CCF-05-14079, and by grants from NSA, PSC-CUNY, Hungarian Research Foundation OTKA, and BSF.

†Supported by NSERC and OTKA grants, and by Sloan Research Fellowship.

¥Supported by NSERC and OTKA grants. 
chip area required for the VLSI circuit layout of a graph. He proved the general lower bound $\operatorname{cr}(G)>c e^{3} / n^{2}-O(n)$, for some $c>0$, which was discovered independently by Ajtai, Chvátal, Newborn, and Szemerédi [ACNS82]. The best known value of the constant, $c=1024 / 31827>0.032$, was found in [PRTT04].

This result was used to deduce the best known lower bound for the number of distinct distances determined by $n$ points in the plane [Sz95], [ST01], [KT04] and upper bound for the number of different ways how a line can split a set of $2 n$ points into two equal parts [D98], and it has some other interesting corollaries [PS98], [PT02], [STT02], [MSSW06], [BCSV07].

It is easy to see that the above bound is tight, apart from the value of the constant. However, as was shown in [PST00], it can be strengthened for some special classes of graphs, e.g., for graphs not containing some fixed, so-called forbidden subgraph. In particular, if $G$ contains no cycle of length four, its crossing number is at least $c^{\prime} e^{4} / n^{3}-O(n)$, for a suitable constant $c^{\prime}>0$.

The order of magnitude of the bounds of Leighton and Ajtai et al. as well as the estimates in [PST00] can be tight only for "nearly regular" graphs. The aim of this note is to establish improved bounds for graphs with irregular degree distributions.

Theorem 1. For any simple graph $G$ on $n$ vertices with vertex degrees $d_{1} \geq$ $d_{2} \geq \ldots \geq d_{n}$ we have

$$
\operatorname{cr}(G) \geq \frac{1}{36000 n} \sum_{i=1}^{n} i d_{i}^{3}-4.01 n^{2}
$$

Another, in most cases somewhat weaker, result of this kind was found independently by Fox and Cs. Tóth [FT06].

The bound in Theorem 1 is tight up to a constant factor, apart from the error term. Note that while the addition of isolated vertices does not change the crossing number of a graph, adding extra zeroes to the degree sequence decreases the lower bound in the theorem (as $n$ increases). Theorem 1 cannot be substantially improved if we restrict our attention to bounds that depend monotonically on the degrees of the vertices. Here we prove the tightness in a bipartite setting.

Theorem 2. For any sequence of integers $n \geq d_{1} \geq d_{2} \geq \ldots \geq d_{n} \geq 0$, there exists a bipartite graph $G$ with $n$ vertices in either vertex class such that the degree sequence in one class is exactly $d_{1}, \ldots, d_{n}$ and

$$
\operatorname{cr}(G) \leq \frac{8}{n} \sum_{i=1}^{n} i d_{i}^{3}
$$

Theorems 1 and 2 show that the minimum crossing number of a graph with degrees $d_{1} \geq \ldots \geq d_{n}$ can be estimated up to a constant factor by the expression $\frac{1}{n} \sum_{i=1}^{n} i d_{i}^{3}$, provided that this minimum exceeds a constant multiple of $n^{2}$. We mention some alternative forms of this estimate. 
As we will see in Section $3, \sum_{i=1}^{n} i d_{i}^{3}$ is always within a constant factor of $\sum_{i=1}^{n} s_{i} d_{i}^{2}$, where $s_{i}=\sum_{j=1}^{i} d_{i}$. In some situations, it is more convenient to use the latter variant.

The expression $\left(\sum_{i=1}^{n} d_{i}^{3 / 2}\right)^{2}$ is also closely related to the sum $\sum_{i=1}^{n} i d_{i}^{3}$. It is more attractive, in the sense that it does not depend on the order of the elements $d_{1}, \ldots, d_{n}$. One can prove that for any sequence of nonnegative reals $d_{1} \geq \ldots \geq d_{n}$, we have

$$
\sum_{i=1}^{n} i d_{i}^{3} \leq\left(\sum_{i=1}^{n} d_{i}^{3 / 2}\right)^{2}=O\left(\log n \sum_{i=1}^{n} i d_{i}^{3}\right) .
$$

The logarithmic factor on the right-hand side cannot be eliminated, as is shown, for example, by the sequence $d_{i}=i^{-2 / 3}$.

Theorems 1 and 2 are proved in Sections 2 and 3. Section 4 contains a direct application of our results.

\section{Imbalanced bipartite Crossing Lemma}

Our computations will be based on the simple observation that in an imbalanced bipartite graph the number of crossings is always larger than the bound guaranteed by the Crossing Lemma.

Lemma 2.1. Let $G(A, B)$ be a bipartite graph with vertex classes $A$ and $B$, and suppose that its number of edges satisfies $e \geq 6 \max (|A|,|B|)$. Then we have

$$
\operatorname{cr}(G(A, B)) \geq \frac{1}{108} \frac{e^{3}}{|A||B|} .
$$

Proof. If $G(A, B)$ is planar, then it follows from Euler's Polyhedral Formula that $e \leq 2(|A|+|B|)-4$, provided that $|A|+|B| \geq 3$. This yields, by induction on $e$, that for not necessarily planar bipartite graphs on at least 3 vertices

$$
\operatorname{cr}(G(A, B)) \geq e-2(|A|+|B|)+4
$$

holds.

Select each vertex of $A$ independently with probability $p_{1}$, and let $A^{\prime}$ denote the set of selected vertices. Analogously, let $B^{\prime}$ be a randomly chosen subset of $B$, whose elements are selected from $B$ independently with probability $p_{2}$. Letting $G\left(A^{\prime}, B^{\prime}\right)$ denote the subgraph of $G(A, B)$ induced by $A^{\prime} \cup B^{\prime},(1)$ implies that

$$
\operatorname{cr}\left(G\left(A^{\prime}, B^{\prime}\right)\right)>e^{\prime}-2\left(\left|A^{\prime}\right|+\left|B^{\prime}\right|\right),
$$

where $e^{\prime}$ stands for the number of edges of $G\left(A^{\prime}, B^{\prime}\right)$. Taking expectations of both sides, we obtain

$$
p_{1}^{2} p_{2}^{2} \operatorname{cr}(G(A, B)) \geq E\left[\operatorname{cr}\left(G\left(A^{\prime}, B^{\prime}\right)\right)\right]
$$




$$
\begin{aligned}
& >E\left[e^{\prime}\right]-2 E\left[\left|A^{\prime}\right|+\left|B^{\prime}\right|\right] \\
& =p_{1} p_{2} e-2\left(p_{1}|A|+p_{2}|B|\right) \\
\operatorname{cr}(G(A, B)) & >\frac{1}{p_{1} p_{2}}\left(e-2\left(\frac{|A|}{p_{2}}+\frac{|B|}{p_{1}}\right)\right) .
\end{aligned}
$$

Setting $p_{1}:=\frac{6|B|}{e}$ and $p_{2}:=\frac{6|A|}{e}$, the result follows.

We can get rid of the assumption $e \geq 6 \max (|A|,|B|)$ in Lemma 2.1 by introducing an error term. For comparison and later reference, we also state the original version of the Crossing Lemma (with the better constant obtained in [PRTT04]).

Lemma 2.2. (i) Let $G$ be a bipartite graph with vertex classes of size $k$ and $n$ with $k \leq n$ and $e$ edges. We have

$$
\operatorname{cr}(G) \geq \frac{1}{108} \frac{e^{3}}{k n}-2 \frac{n^{2}}{k} .
$$

(ii) For an arbitrary simple graph $G$ with $n$ vertices and e edges, we have

$$
\operatorname{cr}(G) \geq \frac{1}{32} \frac{e^{3}}{n^{2}}-2 n .
$$

Proof of (i). Adjusting the constant in the error term, we can achieve that the bound becomes negative and therefore trivially holds when the assumption of Lemma 2.1 is not satisfied.

Proof of Theorem 1. Fix a graph $G$ with vertex set $V(G)=\left\{v_{1}, \ldots, v_{n}\right\}$ with $d\left(v_{i}\right)=d_{i}$ for $i=1, \ldots, n$.

Let $l_{0}=\left\lfloor\log _{2} n\right\rfloor$. For $1 \leq l \leq l_{0}$, consider the pairwise disjoint sets $V_{l}=$ $\left\{v_{i} \mid 2^{l-1} \leq i<2^{l}\right\} \subseteq V(G)$, and let $H_{l}$ denote the subgraph of $G$ induced by $V_{l}$. Let $H_{l}^{\prime} \subseteq G$ be the bipartite subgraph, consisting of all edges of $G$ running between $V_{l}$ and its complement $V(G) \backslash V_{l}$. Finally, let $f_{l}$ and $f_{l}^{\prime}$ denote the number of edges in $H_{l}$ and $H_{l}^{\prime}$.

Set $t_{l}=\sum_{v_{i} \in V_{l}} d_{i}$. Clearly, we have $t_{l}=2 f_{l}+f_{l}^{\prime}$ for every $l$, so that $\max \left(f_{l}, f_{l}^{\prime}\right) \geq t_{l} / 3$. Applying parts (ii) and (i) of Lemma 2.2 to $H_{l}$ and $H_{l}^{\prime}$, respectively, we obtain that

$$
\operatorname{cr}\left(H_{l}\right) \geq \frac{f_{l}^{3}}{2^{2 l+3}}-2^{l} \text { and } \operatorname{cr}\left(H_{l}^{\prime}\right) \geq \frac{f_{l}^{\prime 3}}{54 \cdot 2^{l} n}-\frac{4 n^{2}}{2^{l}} .
$$

This yields

$$
\max \left(\operatorname{cr}\left(H_{l}\right), \operatorname{cr}\left(H_{l}^{\prime}\right)\right) \geq \frac{t_{l}^{3}}{1500 \cdot 2^{l} n}-\frac{4 n^{2}}{2^{l}} .
$$

The graphs $H_{l}$ and $H_{l}^{\prime}\left(1 \leq l \leq l_{0}\right)$ have the property that every edge belongs to at most two of them. Thus, we have

$$
\operatorname{cr}(G) \geq \sum_{l=1}^{l_{0}} \frac{\operatorname{cr}\left(H_{l}\right)+\operatorname{cr}\left(H_{l}^{\prime}\right)}{2} \geq \frac{1}{3000 n} \sum_{l=1}^{l_{0}} \frac{t_{l}^{3}}{2^{l}}-4 n^{2} .
$$


In order to prove Theorem 1, it is enough to show that the above bound exceeds the one stated in the theorem. It follows from the fact that the sequence $d_{1}, d_{2}, \ldots$ is monotone decreasing that

$$
t_{l}=\sum_{i=2^{l-1}}^{2^{l}-1} d_{i} \geq 2^{l-1} d_{2^{l}},
$$

for $1 \leq l \leq l_{0}$.

Consider the partial sum

$$
D_{l}=\sum_{i=2^{l}}^{\max \left(2^{l+1}-1, n\right)} i d_{i}^{3} .
$$

Obviously, we have

$$
\sum_{l=1}^{l_{0}} D_{l}=\sum_{i=2}^{n} i d_{i}^{3}=\sum_{i=1}^{n} i d_{i}^{3}-d_{1}^{3} .
$$

Using again the monotonicity of the degree sequence, we conclude that

$$
D_{l} \leq 3 \cdot 2^{2 l-1} d_{2^{l}}^{3} \leq 12 \frac{t_{l}^{3}}{2^{l}},
$$

so that

$$
\begin{aligned}
\operatorname{cr}(G) & \geq \frac{1}{3000 n} \sum_{l=1}^{l_{0}} \frac{t_{l}^{3}}{2^{l}}-4 n^{2} \\
& \geq \frac{1}{36000 n} \sum_{l=1}^{l_{0}} D_{l}-4 n^{2} \\
& \geq \frac{1}{36000 n} \sum_{i=1}^{n} i d_{i}^{3}-4.01 n^{2} .
\end{aligned}
$$

\section{A bipartite construction}

For the proof of Theorem 2, we need the following technical lemma.

Lemma 3.1 Let $d_{1}, \ldots, d_{n}$ be a sequence of non-negative reals. For $1 \leq i \leq n$, let $s_{i}=\sum_{j=1}^{i} d_{j}$. We have

$$
\sum_{i=1}^{n} s_{i} d_{i}^{2} \leq 4 \sum_{i=1}^{n} i d_{i}^{3} .
$$

Proof. First, notice that

$$
2 \sum_{j=1}^{i} s_{j} d_{j}=2 \sum_{j=1}^{i} \sum_{k=1}^{j} d_{j} d_{k}=s_{i}^{2}+\sum_{j=1}^{i} d_{j}^{2} .
$$


Therefore, we have

$$
s_{i} \leq \frac{2}{s_{i}} \sum_{j=1}^{i} s_{j} d_{j}
$$

for all $i$.

Introducing the notation $A=\sum_{i=1}^{n} s_{i} d_{i}^{2}$ and $B=\sum_{i=1}^{n} i d_{i}^{3}$, in view of the last inequality, we have

$$
\begin{gathered}
A \leq 2 \sum_{i=1}^{n} \frac{d_{i}^{2}}{s_{i}} \sum_{j=1}^{i} s_{j} d_{j}, \\
\frac{1}{2} A-B \leq \sum_{i=1}^{n} d_{i}^{2} \sum_{j=1}^{i}\left(\frac{s_{j}}{s_{i}} d_{j}-d_{i}\right) .
\end{gathered}
$$

Using the estimate $d_{i}\left(x-d_{i}\right) \leq x^{2} / 4$ for $x=\left(s_{j} / s_{i}\right) d_{j}$, and switching the order of the summations, we obtain

$$
\frac{1}{2} A-B \leq \sum_{i=1}^{n} \frac{d_{i}}{4} \sum_{j=1}^{i}\left(\frac{s_{j}}{s_{i}} d_{j}\right)^{2}=\frac{1}{4} \sum_{j=1}^{n} s_{j}^{2} d_{j}^{2} \sum_{i=j}^{n} \frac{d_{i}}{s_{i}^{2}}
$$

Notice that

$$
\frac{d_{i}}{s_{i}^{2}} \leq \frac{1}{s_{i-1}}-\frac{1}{s_{i}}
$$

so that for every $i>j$ we have

$$
\sum_{i=j}^{n} \frac{d_{i}}{s_{i}^{2}} \leq \frac{d_{j}}{s_{j}^{2}}+\frac{1}{s_{j}}
$$

This yields

$$
\frac{1}{2} A-B \leq \frac{1}{4} \sum_{j=1}^{n}\left(s_{j} d_{j}^{2}+d_{j}^{3}\right)=\frac{1}{4}\left(A+\sum_{j=1}^{n} d_{j}^{3}\right),
$$

and, by rearranging the terms, $A \leq 4 B+\sum_{i=1}^{n} d_{i}^{3}$.

To get rid of the error term of $C=\sum_{i=1}^{n} d_{i}^{3}$, we simply apply the last inequality to the sequence $\left(d_{i}^{\prime}\right)_{i=1}^{2 n}$ obtained from $\left(d_{i}\right)_{i=1}^{n}$ by repeating each term twice. The corresponding sums for this sequence are $A^{\prime}=4 A-C, B^{\prime}=4 B-C$, and $C^{\prime}=2 C$. We obtain $A^{\prime} \leq 4 B^{\prime}+C^{\prime}$, which implies $A \leq 4 B$, as claimed.

We suspect that Lemma 3.1 remains true with the constant 4 replaced by 3 . However, as is shown by the sequence $d_{i}=i^{-2 / 3}$, the claim is certainly false with any constant smaller than 3 .

Proof of Theorem 2. We construct $G$ together with a straight-line drawing that will demonstrate the upper bound on the crossing number of $G$. Let $\left\{v_{1}, \ldots, v_{n}\right\}$ and $\left\{w_{1}, \ldots, w_{n}\right\}$ be the two vertex classes of $G$. Pick a line $\ell$, and place the points $w_{1}, \ldots, w_{n}$ on $\ell$ in this order, from left to right. The positions of the vertices $v_{1}, \ldots, v_{n}$ will be determined one by one, so that no $v_{i}$ lies on $\ell$ 
or on any previously drawn edge, and each $v_{i}$ is connected by a segment to the prescribed number $d_{i}$ of points $w_{j}$.

Place $v_{1}$ at any point that does not belong to $\ell$, and connect it to the vertices $w_{1}, \ldots, w_{d_{1}}$. Now let $i>1$, and assume that the position of all vertices $v_{1}, \ldots, v_{i-1}$ has already been fixed. If $d_{i}=0$, then $v_{i}$ is an isolated vertex and it can be placed anywhere outside of $\ell$ and the previously drawn edges. If $d_{i}>0$, then let $w_{j}$ denote the last vertex (that is, the one with the highest index) incident to $v_{i-1}$.

If $n-j \geq d_{i}$, connect $v_{i}$ to $w_{j+1}, w_{j+2}, \ldots, w_{j+d_{i}}$. If $n-j<d_{i}$, connect $v_{i}$ to $w_{1}, \ldots, w_{d_{i}}$. Place $v_{i}$ at a point (not belonging to $\ell$ or to any previously drawn edge) which lies so close to one of its neighbors that any edge incident to $v_{i}$ can cross only those edges that are incident to a neighbor of $v_{i}$. As the neighbors of $v_{i}$ are consecutive points on $\ell$, this can indeed be achieved.

Obviously, the resulting geometric graph is bipartite and the degrees of its vertices satisfy $d\left(v_{i}\right)=d_{i}$. It remains to estimate the number of crossings. Fix a vertex $v_{i}$ and consider its neighbors $w_{j}$. Let $s_{i}$ denote the maximum degree of a neighbor of $v_{i}$ in the graph induced by the previously processed vertices $v_{1}, \ldots, v_{i-1}$. In other words, $s_{i}$ is the number of times our drawing algorithms had to "start over" at $w_{1}$ before processing $v_{i}$. The numbers $d_{i}$ were listed in decreasing order, therefore in each round at least $n / 2$ edges were drawn. Thus, the total number of edges drawn before processing $v_{i}$ is $\sum_{j=1}^{i-1} d_{j} \geq s_{i} n / 2$, so that

$$
s_{i} \leq \frac{2}{n} \sum_{j=1}^{i-1} d_{j} .
$$

According to the drawing rules, the introduction of each $v_{i}$ creates at most $s_{i} d_{i}^{2}$ new crossings. Thus, we have $\operatorname{cr}(G) \leq \sum_{i=1}^{n} s_{i} d_{i}^{2}$. Applying Lemma 3.1, we obtain

$$
\operatorname{cr}(G) \leq \frac{8}{n} \sum_{i=1}^{n} i d_{i}^{3}
$$

as required.

\section{Applications and concluding remarks}

Given a set $P$ of $2 n$ points in general position in the plane, two elements of $P$ form a halving pair if the line connecting them divides $P$ into two parts of equal cardinality [L71]. The best known upper bound for the number of halving pairs, $O\left(n^{4 / 3}\right)$, was established by Dey [D98]. His result was strengthened by Andrzejak, Aronov, Har-Peled, Seidel, and Welzl [AAHSW98, AW03], as follows. Define the halving-edge graph $H(P)$ of $P$, as a geometric graph on the vertex set $P$, where two vertices are connected by a straight-line segment (edge) if and only if they form a halving pair. For the degree sequence $d_{1}, d_{2}, \ldots, d_{2 n}$ 
of the vertices of $H(P)$, Andrzejak et al. found the beautiful formula

$$
\kappa(H(P))+\sum_{i=1}^{2 n}\left(\begin{array}{c}
\left(d_{i}+1\right) / 2 \\
2
\end{array}\right)=\left(\begin{array}{l}
n \\
2
\end{array}\right),
$$

where $\kappa(H(P))$ denotes the number of crossing pairs of edges of $H(P)$. It follows that $\kappa(H(P))<n^{2} / 2$, and combining this bound with the Crossing Lemma, we immediately obtain Dey's result. Note that here the contribution of the sum of the squares of the degrees is negligible, therefore we gain no information on the degree distribution. By Theorem 1, we have

Corollary 3. For the degree sequence $d_{1} \geq d_{2} \geq \ldots \geq d_{2 n}$ of the halving-edge graph $H(P)$ of a $2 n$-element point set $P$ in general position in the plane, we have

$$
\sum_{i=1}^{2 n} i d_{i}^{3} \leq C n^{3}
$$

where $C$ is a positive constant.

This inequality is a strengthening of Dey's bound. It implies that if there exists a point set with $\Omega\left(n^{4 / 3}\right)$ halving pairs, then its halving-edge graph must have a fairly even degree distribution. Analogously, we can generalize other applications of the Crossing Lemma, and conclude that the resulting estimates cannot be asymptotically tight, unless the degrees of the vertices in the corresponding graph are roughly the same.

\section{References}

[ACNS82] M. Ajtai, V. Chvátal, M. Newborn, and E. Szemerédi, Crossing-free subgraphs, in: Theory and Practice of Combinatorics, North-Holland Math. Stud. 60, North-Holland, Amsterdam-New York, 1982, 9-12.

[AAHSW98] A. Andrzejak, B. Aronov, S. Har-Peled, R. Seidel, and E. Welzl, Results on k-sets and j-facets via Continuous Motions, in: Proc. 14th Ann. ACM Symposium on Computational Geometry (SOCG), 1998, 192-199.

[AW03] A. Andrzejak and E. Welzl, Halving point sets, in: Proc. Internat. Congress of Mathematicians (Berlin, 1998), Doc. Math. 1998, Extra Vol. III, 471-478.

[BCSV07] D. Bokal, E. Czabarka, L. A. Székely, and I. Vrt́o, Graph minors and the crossing number of graphs (6th Czech-Slovak International Symposium on Combinatorics, Graph Theory, Algorithms and Applications), Electronic Notes in Discrete Math. 28 (2007), 169-175.

[D98] T. K. Dey, Improved bounds for planar $k$-sets and related problems, Discrete Computat. Geom. 19 (1998), 373-382. 
[FPT07] J. Fox, J. Pach, and Cs. T. Tóth, A bipartite strengthening of the Crossing Lemma, in: Graph Drawing (S.-H. Hong, T. Nishizeki, eds.), Lecture Notes in Computer Science 4875, Springer-Verlag, Berlin, 2007, 13-24.

[FT06] J. Fox and Cs. D. Tóth, On the decay of crossing numbers, in: Graph Drawing (Karlsruhe, 2006), Lecture Notes in Computer Science 4372, Springer-Verlag, Berlin, 2007, 174-183. Also in: J. Combin. Theory Ser. B (2007), to appear.

[GJ83] M. R. Garey and D. S. Johnson, Crossing number is NP-complete, SIAM J. Alg. Disc. Meth. 4 (1983), 312-316.

[KT04] N. H. Katz and G. Tardos, A new entropy inequality for the Erdős distance problem, in: Towards a Theory of Geometric Graphs, Contemp. Math. 342, Amer. Math. Soc., Providence, RI, 2004, 119-126.

[L83] F. T. Leighton, Complexity Issues in VLSI, MIT Press, Cambridge, 1983.

[L71] L. Lovász, On the number of halving lines, Ann. Univ. Sci. Budapest, Eötvös, Sect. Math. 14 (1971), 107-108.

[MSSW06] J. Matoušek, M. Sharir, Micha; S. Smorodinsky, and U. Wagner, $k$-sets in four dimensions, Discrete Comput. Geom. 35 (2006), 177-191.

[PRTT04] J. Pach, R. Radoičić, G. Tardos, and G. Tóth, Improving the Crossing Lemma by finding more crossings in sparse graphs, in: 20th ACM Symp. Comput. Geom., ACM Press, New York, 2004, 68-75. Also in: Discrete Comput. Geom. 36 (2006), 527-552.

[PS98] J. Pach and M. Sharir, On the number of incidences between points and curves, Combin. Probab. Comput. 7 (1998), 121-127.

[PST00] J. Pach, J. Spencer, and G. Tóth, New bounds on crossing numbers, Discrete Computat. Geom. 24 (2000), 623-644.

[PT02] J. Pach and G. Tardos, Isosceles triangles determined by a planar point set, Graphs Combin. 18 (2002), 769-779.

[PT97] J. Pach and G. Tóth, Graphs drawn with few crossings per edge, Combinatorica 17 (1997), 427-439.

[R04] I. Z. Ruzsa, A problem on restricted sumsets, in: Towards a Theory of Geometric Graphs, Contemp. Math. 342, Amer. Math. Soc., Providence, RI, 2004, 245-248.

[STT02] J. Solymosi, G. Tardos, and Cs. D. Tóth, The $k$ most frequent distances in the plane, Discrete Comput. Geom. 28 (2002), 639-648.

[ST01] J. Solymosi and Cs. D. Tóth, Distinct distances in the plane, Discrete Comput. Geom. 25 (2001), 629-634. 
[Sz95] L. Székely, Crossing numbers and hard Erdős problems in discrete geometry, Combin., Probab., and Comp., 6 (1997), 353-358. 